\title{
FIXED-POINT ITERATIVE SWEEPING METHODS FOR STATIC HAMILTON-JACOBI EQUATIONS*
}

\author{
YONG-TAO ZHANG ${ }^{\dagger}, \mathrm{HONG}^{-K A I} \mathrm{ZHAO}^{\ddagger}$, AND SHANQIN CHEN $\S$ \\ Dedicated to Professor Bjorn Engquist on the occasion of his 60th birthday
}

\begin{abstract}
Fast sweeping methods utilize the Gauss-Seidel iterations and alternating sweeping strategy to achieve the fast convergence for computations of static Hamilton-Jacobi equations. They take advantage of the properties of hyperbolic PDEs and try to cover a family of characteristics of the corresponding Hamilton-Jacobi equation in a certain direction simultaneously in each sweeping order. The time-marching approach to steady state calculation is much slower than the fast sweeping methods due to the CFL condition constraint. But this kind of fixed-point iterations as timemarching methods have explicit form and do not involve inverse operation of nonlinear Hamiltonian. So it can solve general Hamilton-Jacobi equations using any monotone numerical Hamiltonian and high order approximations easily. In this paper, we adopt the Gauss-Seidel idea and alternating sweeping strategy to the time-marching type fixed-point iterations to solve the static Hamilton-Jacobi equations. Extensive numerical examples verify at least a $2 \sim 5$ times acceleration of convergence even on relatively coarse grids. The acceleration is even more when the grid is further refined. Moreover the Gauss-Seidel philosophy and alternating sweeping strategy improves the stability, i.e., a larger CFL number can be used. Also the computational cost is exactly the same as the time-marching scheme at each time step.
\end{abstract}

Key words. fast sweeping methods, Jacobi iteration, Gauss-Seidel iteration, static HamiltonJacobi equations, Eikonal equations

AMS subject classifications. Primary, 65N99; Secondary, 35L60

1. Introduction. The steady state calculations of Hamilton-Jacobi (H-J) equations appear in many applications, such as optimal control, differential games, image processing and computer vision, geometric optics, etc. The general form of steady H-J equations is

$$
\left\{\begin{array}{l}
H\left(\phi_{x_{1}}, \cdots, \phi_{x_{d}}, x\right)=0, \quad x \in \Omega \backslash \Gamma, \\
\phi(x)=g(x), \quad x \in \Gamma \subset \Omega,
\end{array}\right.
$$

where $\Omega$ is a computational domain in $R^{d}$ and $\Gamma$ is a subset of $\Omega$. The Hamiltonian $H$ is a nonlinear Lipschitz continuous function. The concept of viscosity solutions for Hamilton-Jacobi equations was introduced in [7].

For the general H-J equations (1.1), a straightforward way is the time marching approach which turns it into a corresponding time dependent problem and evolves it

\footnotetext{
*Received November 9, 2005; accepted for publication May 26, 2006.

$\dagger$ Department of Mathematics, University of California, Irvine, CA 92697-3875, USA (zyt@math. uci.edu). Current Address: Department of Mathematics, University of Notre Dame, 255 Hurley Hall, Notre Dame, IN 46556-4618, USA (yzhang10@nd.edu).

${ }^{\ddagger}$ Department of Mathematics, University of California, Irvine, CA 92697-3875, USA (zhao@ math.uci.edu). The research is partially supported by ONR grant N00014-02-1-0090, DARPA grant N00014-02-1-0603, the Sloan Foundation and NSF grant DMS0513073.

$\S$ Department of Mathematics, University of California, Irvine, CA 92697-3875, USA (shanqin@ math.uci.edu). Current Address: Department of Mathematical Sciences, Indiana University South Bend, 1700 Mishawaka Ave., P.O. Box 7111, South Bend, IN 46634, USA (chen39@iusb.edu).
} 
to steady state:

$$
\left\{\begin{array}{l}
\phi_{t}+H\left(\phi_{x_{1}}, \cdots, \phi_{x_{d}}, x\right)=0, \quad x \in \Omega \backslash \Gamma, \\
\phi(x, t)=g(x), \quad x \in \Gamma \subset \Omega, \\
\phi(x, 0)=\phi_{0}(x) .
\end{array}\right.
$$

Numerical schemes for time dependent Hamilton-Jacobi equations (1.2) are well developed, even for high order schemes on both structured and unstructured meshes $[23,14,38,13,22,6,15,20,24,1,2,5,4]$; see a recent review on high order numerical methods for time dependent H-J equations by Shu [33]. In [21], Osher provides a natural link between steady and time-dependent Hamilton-Jacobi equations by using the level-set idea. The zero-level set of the viscosity solution $\psi$ of the time-dependent H-J equation

$$
\psi_{t}+H\left(\psi_{x_{1}}, \cdots, \psi_{x_{d}}, x\right)=0
$$

at time $t$ is the set of $x$ such that $\phi(x)=t$ of (1.1), where the Hamiltonian $H$ is homogeneous of degree one. In the control framework, a semi-Lagrangian scheme is obtained for Hamilton-Jacobi equations by discretizing in time using the dynamic programming principle $[9,10]$. Another approach to obtain a "time" dependent H-J equation from the steady H-J equation is using the so called paraxial formulation in which a preferred spatial direction is assumed in the characteristic propagation $[11,8,16,26,27]$. The convergence to steady state of the solution in the entire domain may be slow due to finite speed of propagation and CFL condition for the discrete time step size. The other class of numerical methods for steady state calculations of H-J equations is to treat the problem as a stationary boundary value problem: discretize the problem into a system of nonlinear equations and design an efficient numerical algorithm to solve the system. Among such methods are the fast marching method $[37,30,12,31,32]$ and the fast sweeping method $[3,41,36,40,17,18,39,28]$. The main idea of the fast sweeping method is to use upwind finite differences and GaussSeidel iterations with alternating direction sweepings. Dividing all characteristics into a finite number of groups according to their directions, the fast sweeping method follows the causality along characteristics in a parallel way and covers a group of characteristics simultaneously in each Gauss-Seidel iteration with a specific sweeping ordering. More details of fast sweeping methods will be reviewed in the next section.

While the fast sweeping method can be optimal in the sense that a finite number of iterations is needed [40], so that the complexity of the algorithm is $O(N)$ for a total of $N$ grid points; i.e., the number of iterations is independent of the grid size, it has the framework of implicit schemes. When the high order accuracy version of fast sweeping methods are explored, this implicit framework generates nonlinear equations which incorporate both the nonlinearity of high order schemes and nonlinearity of Hamiltonian. Hence it is not straightforward to solve it directly. A semi-implicit way was introduced to simplify this procedure in [39]. On the other hand, although the time marching approach is slow to converge to steady states, it is explicit and has a simple form of the fixed-point iteration scheme. So it is straightforward to apply high order approximations and different numerical Hamiltonian for the general Hamilton-Jacobi equations.

In this paper, we generalized the idea in [39] to the time marching approach and explored a way to accelerate the convergence to steady states for the time marching approach. The Gauss-Seidel idea and alternating sweeping strategy are adopted to 
the time marching approach to accelerate its convergence to steady states without any additional computational cost. Numerical experiments are performed to verify this acceleration.

In most part of the paper, we use the standard isotropic Eikonal equation

$$
\left\{\begin{array}{l}
|\nabla \phi(x)|=f(x), \quad x \in \Omega \backslash \Gamma, \\
\phi(x)=g(x), \quad x \in \Gamma \subset \Omega,
\end{array}\right.
$$

where $f(x)$ is a positive function, to be the representation of static H-J equations. The classical shape-from-shading problems $[19,29,14,13]$ are chosen to be the numerical examples. A more complicated example will also be presented to test our methods at the end.

In Section 2, we review the fast sweeping methods [40] for the eikonal equation (1.4). In Section 3, the idea of fast sweeping methods is applied to the time-marching type fixed-point iteration to accelerate its convergence to steady states. Numerical studies are performed to verify the faster convergence speed than the usual timemarching approach. Concluding remarks are given in Section 4.

2. Review of fast sweeping methods. In this section, we review the first order Godunov fast sweeping methods in [40]. We consider the two dimensional problems for simplicity. We first construct a Cartesian grid $\Omega_{h}=\{(i, j), 1 \leq i \leq I, 1 \leq j \leq J\}$ covering the computational domain $\Omega$, denote a grid point by $(i, j)$ and the viscosity numerical solution of (1.1) at the grid point by $\phi_{i, j}$. Then discretize the steady H-J equation (1.1) directly by a monotone numerical Hamiltonian $\hat{H}$ [23]:

$$
\left\{\begin{array}{l}
\hat{H}\left(\left(\phi_{x}\right)_{i, j}^{-},\left(\phi_{x}\right)_{i, j}^{+},\left(\phi_{y}\right)_{i, j}^{-},\left(\phi_{y}\right)_{i, j}^{+}\right)=f_{i, j}, \quad(i, j) \in \Omega_{h} \backslash \Gamma_{h}, \\
\phi_{i, j}=g_{i, j}, \quad(i, j) \in \Gamma_{h} \subset \Omega_{h},
\end{array}\right.
$$

where $f_{i, j}$ and $g_{i, j}$ denote the value of $f(x)$ and $g(x)$ at grid point $(i, j)$ respectively, $\left(\phi_{x}\right)_{i, j}^{-}$denotes the approximation of $\phi_{x}$ at the grid point $(i, j)$ when the wind "blows" from the left to the right, and $\left(\phi_{x}\right)_{i, j}^{+}$denotes the approximation of $\phi_{x}$ at the grid point $(i, j)$ when the wind "blows" from the right to the left; similar for $\left(\phi_{y}\right)_{i, j}^{-}$and $\left(\phi_{y}\right)_{i, j}^{+}$.

Because of the nonlinearity of general Hamiltonian $H$, the $I \times J$ nonlinear system (2.1) is usually very complicated to solve, especially when the high order nonlinear approximations for derivatives $\phi_{x}, \phi_{y}$ are applied [39]. So an efficient and robust algorithm to solve (2.1) is very desirable.

To study the convergence history and compare the convergence speed for different iterative algorithms, we calculate the discrete $L^{1}$ norm of the nonlinear residue at every iteration step for each algorithm. The nonlinear residue at the $n$th iteration step $r^{(n)}$ is

$$
r_{i, j}^{(n)}=f_{i, j}-\hat{H}^{(n)}\left(\left(\phi_{x}\right)_{i, j}^{-},\left(\phi_{x}\right)_{i, j}^{+} ;\left(\phi_{y}\right)_{i, j}^{-},\left(\phi_{y}\right)_{i, j}^{+}\right), \quad 1 \leq i \leq I, 1 \leq j \leq J .
$$

The notation $\hat{H}^{(n)}\left(\left(\phi_{x}\right)_{i, j}^{-},\left(\phi_{x}\right)_{i, j}^{+} ;\left(\phi_{y}\right)_{i, j}^{-},\left(\phi_{y}\right)_{i, j}^{+}\right)$denotes the left hand side of nonlinear system $(2.1) \hat{H}\left(\left(\phi_{x}\right)_{i, j}^{-},\left(\phi_{x}\right)_{i, j}^{+},\left(\phi_{y}\right)_{i, j}^{-},\left(\phi_{y}\right)_{i, j}^{+}\right)$taking value at $\phi^{(n)}$, where $\phi^{(n)}$ is the numerical solution after the $n$th iteration. We consider an iterative method is convergent if

$$
\left\|r^{(n)}\right\|_{L^{1}} \leq \varepsilon
$$


where $\varepsilon$ is chosen to be $10^{-11}$ in our numerical experiments.

On the other hand, we also care about the accuracy of the algorithm. So we calculate the $L^{1}$ norm of the error $e^{(n)}$ between the exact viscosity solution $\phi^{\text {exact }}$ and numerical solution $\phi^{(n)}$ for every iteration step. The $e^{(n)}$ at each grid point is

$$
e_{i, j}^{(n)}=\phi_{i, j}^{\text {exact }}-\phi_{i, j}^{(n)} .
$$

We take $d=2$ in (1.4):

$$
\left\{\begin{array}{l}
\sqrt{\phi_{x}^{2}+\phi_{y}^{2}}=f(x, y), \quad(x, y) \in \Omega \subset R^{2}, \\
\phi(x, y)=g(x, y), \quad(x, y) \in \Gamma \subset \Omega
\end{array}\right.
$$

A first-order Godunov upwind difference scheme is used to discretize the PDE $(2.5)$ in $[40]$ :

$$
\left[\left(\frac{\phi_{i, j}-\phi_{i, j}^{(x \min )}}{h}\right)^{+}\right]^{2}+\left[\left(\frac{\phi_{i, j}-\phi_{i, j}^{(y \min )}}{h}\right)^{+}\right]^{2}=f_{i, j}^{2},
$$

where $\phi_{i, j}^{(x m i n)}=\min \left(\phi_{i-1, j}, \phi_{i+1, j}\right), \phi_{i, j}^{(y m i n)}=\min \left(\phi_{i, j-1}, \phi_{i, j+1}\right)$ and

$$
(x)^{+}= \begin{cases}x, & x>0 \\ 0, & x \leq 0 .\end{cases}
$$

One sided difference is used at the boundary of the computational domain.

Initialization: according to the boundary condition $\phi(x, y)=g(x, y)$ for $(x, y) \in \Gamma$, assign exact values or interpolated values at grid points in or near $\Gamma$. These values are fixed during iterations. Assign large positive values at all other grid points. These values will be updated later.

Gauss-Seidel iterations with alternating sweeping orderings: At each grid $x_{i, j}$ whose value is not fixed during the initialization, compute the solution, denoted by $\bar{u}$, of (2.6) from the current values of its neighbors $\phi_{i \pm 1, j}, \phi_{i, j \pm 1}$ and then update $\phi_{i, j}$ to be the smaller one between $\bar{u}$ and its current old value, i.e., $\phi_{i, j}^{\text {new }}=\min \left(\phi_{i, j}^{\text {old }}, \bar{u}\right)$. We sweep the whole domain with four alternating directions repeatedly,

$$
\begin{aligned}
& \text { (1) } i=1: I, j=1: J ; \\
& \text { (2) } i=I: 1, j=1: J \\
& \text { (3) } i=I: 1, j=J: 1 ; \\
& \text { (4) } i=1: I, j=J: 1 .
\end{aligned}
$$

The unique solution to the equation (2.6) is

$$
\phi_{i, j}^{\text {new }}=\left\{\begin{array}{l}
\min \left(\phi_{i, j}^{(x \sin )}, \phi_{i, j}^{(y m i n)}\right)+f_{i, j} h, \quad\left|\phi_{i, j}^{(\text {xmin })}-\phi_{i, j}^{(y m i n)}\right| \geq f_{i, j} h, \\
\frac{\phi_{i, j}^{(x m i n)}+\phi_{i, j}^{(y m i n)}+\sqrt{2 f_{i, j}^{2} h^{2}-\left(\phi_{i, j}^{(x m i n)}-\phi_{i, j}^{(y m i n)}\right)^{2}}}{2}, \quad \text { otherwise. }
\end{array}\right.
$$



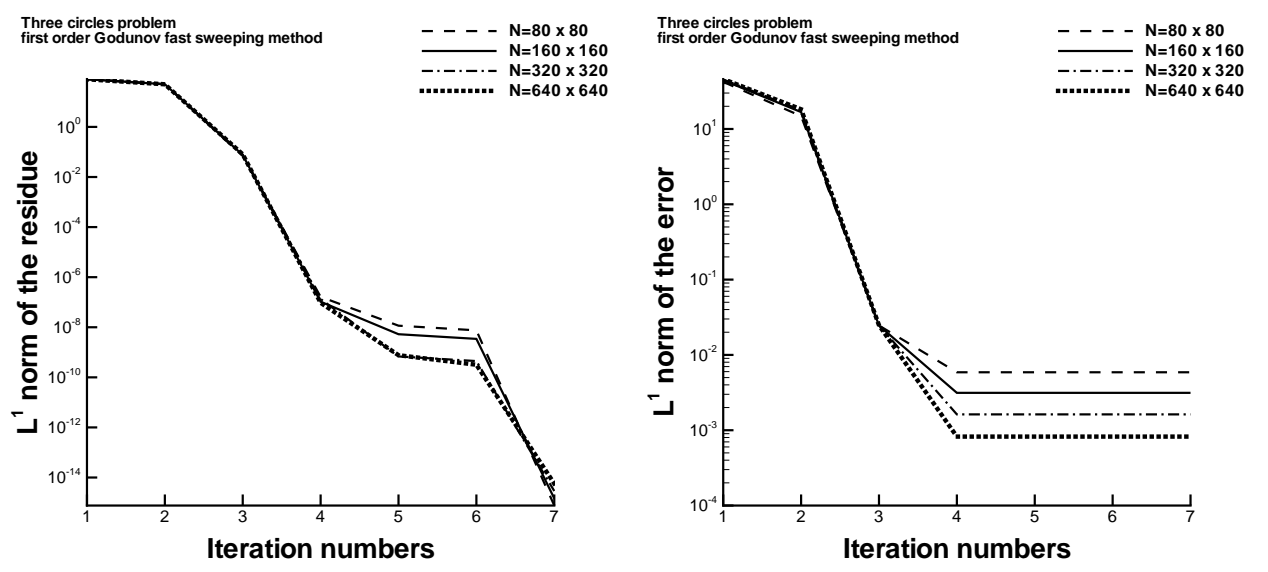

FIG. 2.1. Three circles problem, the first order Godunov fast sweeping method. Mesh refinement study. Left: residue; right: error.

The computational complexity of the algorithm is $O(N)$ for a total of $N$ grid points and the number of iterations is independent of grid size. We use a simple example, the three circles problem to verify this. In equation (1.4), we take $f(x)=$ $1, g(x)=0, \Omega=[-1,1] \times[-1,1]$, and $\Gamma$ is three circles with same radius 0.125 and centers $(-0.5,0.5),(0.5,0.5)$ and $(0,-0.5)$ respectively. The exact solution to this problem is the distance function to these three circles. We use the first order Godunov fast sweeping method in [40], which is reviewed above, to solve this problem. In Figure (2.1), we plot the $L^{1}$ norm of the residue (2.2) and the $L^{1}$ norm of the error (2.4). From the left picture of Figure (2.1), we can see that the residue of the first order Godunov fast sweeping method converges to machine zero in 7 iterations for all of different meshes in this example. The first order accuracy is verified in the right picture of Figure (2.1). It is shown in [40] that for distance function in $n$ dimensions the numerical solution reaches first order accuracy after $2^{n}$ iterations even though a few more iterations may be needed for the iterations to converge. This is also demonstrated by this example. The three-dimensional plot and the contour plot of the numerical solution after 7 iterations on a $160 \times 160$ mesh are plotted in Figure $(2.2)$.

To show the importance of the alternating direction sweepings, we eliminate the alternating direction sweepings in previous computations, and the convergence history is plotted in Figure (2.3) for different meshes. Since there are characteristics whose directions are against the fixed sweeping order, information has to be passed one grid by one grid during the iterations for those characteristics. That is why the number of iterations is proportional to the number of grids in each direction, which is clearly demonstrated by the numerical example. For convex Hamiltonian with upwind numerical scheme and Gauss-Seidel iterations alternating sweeping direction is crucial. However, it may be difficult to have a true Gauss-Seidel for more complicated Hamiltonian, since the non-linear equation corresponding to the numerical Hamiltonian may be difficult to solve at each grid point.

3. Fixed-point iterative sweeping methods. The explicit time marching schemes for (1.2), using the simple forward Euler as the time discretization can be 

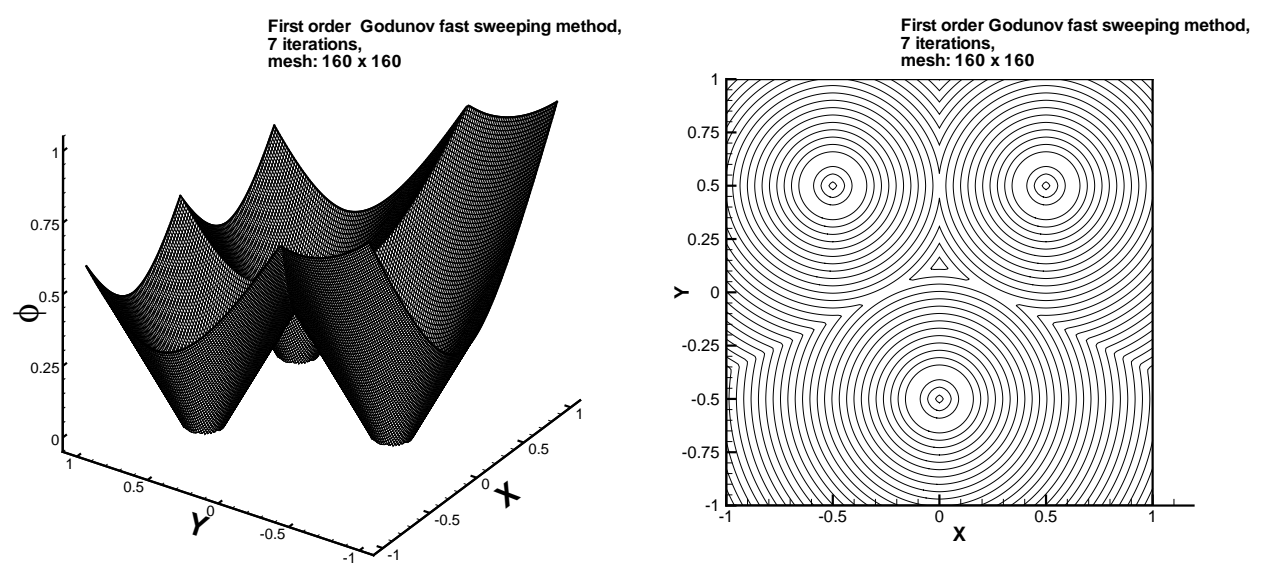

FIG. 2.2. Three circles problem, the first order Godunov fast sweeping method. $160 \times 160$ mesh. Left: three-dimensional view; right: contour lines view, 30 equally spaced contour lines from $\phi=0$ to $\phi=1$.
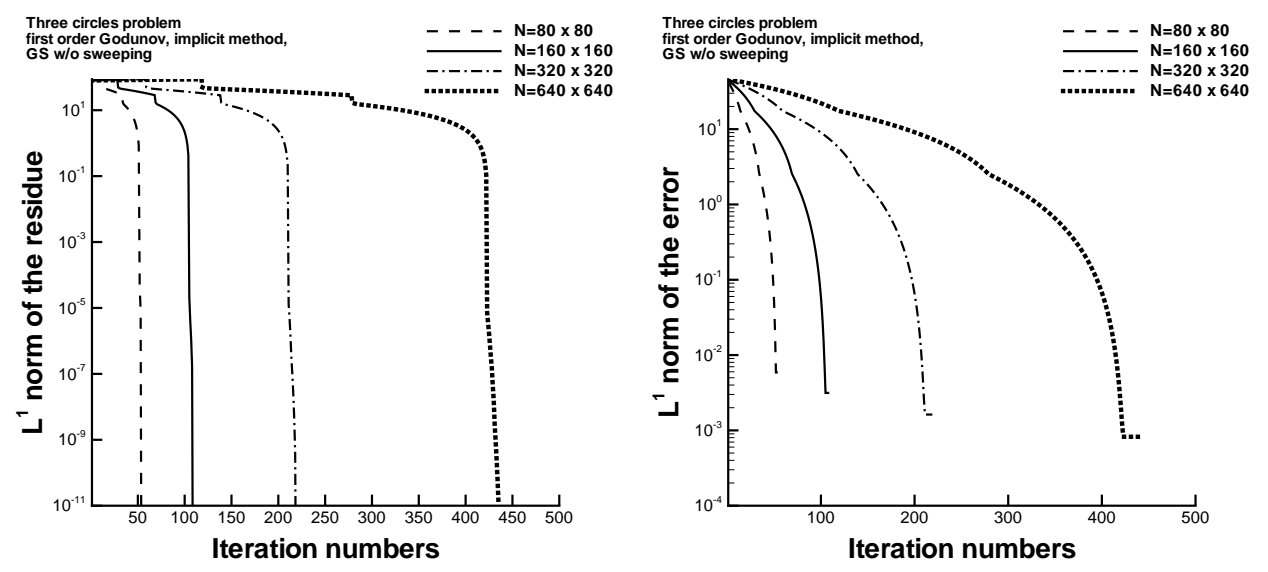

FIG. 2.3. Three circles problem, the first order Godunov method, using Gauss-Seidel iterations but without alternating direction sweepings. Mesh refinement study. Left: residue; right: error.

written in the form

$$
\phi_{i, j}^{n+1}=\phi_{i, j}^{n}+\Delta t\left[f_{i, j}-\hat{H}\left(\left(\phi_{x}\right)_{i, j}^{-},\left(\phi_{x}\right)_{i, j}^{+} ;\left(\phi_{y}\right)_{i, j}^{-},\left(\phi_{y}\right)_{i, j}^{+}\right)\right],
$$

where

$$
\Delta t=\gamma\left(\frac{1}{\frac{\alpha_{x}}{h_{x}}+\frac{\alpha_{y}}{h_{y}}}\right),
$$

$\gamma$ is the CFL number, and

$$
\alpha_{x}=\max _{\substack{A \leq u \leq B \\ C \leq v \leq D}}\left|H_{1}(u, v)\right|, \quad \alpha_{y}=\max _{\substack{A \leq u \leq B \\ C \leq v \leq D}}\left|H_{2}(u, v)\right| .
$$

Here $H_{i}(u, v)$ is the partial derivative of $H$ with respect to the $i$-th argument, or the Lipschitz constant of $H$ with respect to the $i$-th argument. [ $A, B]$ is the value range 
for $\phi_{x}^{ \pm}$, and $[C, D]$ is the value range for $\phi_{y}^{ \pm}$. For the isotropic Eikonal equation (1.4), it is easy to show that $\alpha_{x}=\alpha_{y}=1$.

From the point of view of iterative methods, the scheme (3.1) can be considered as a fixed-point iterative method

$$
\phi_{i, j}^{\text {new }}=\phi_{i, j}^{\text {old }}+\gamma\left(\frac{1}{\frac{\alpha_{x}}{h_{x}}+\frac{\alpha_{y}}{h_{y}}}\right)\left[f_{i, j}-\hat{H}\left(\left(\phi_{x}\right)_{i, j}^{-},\left(\phi_{x}\right)_{i, j}^{+} ;\left(\phi_{y}\right)_{i, j}^{-},\left(\phi_{y}\right)_{i, j}^{+}\right)\right] .
$$

$\phi_{i, j}^{n e w}$ denotes the to-be-updated numerical solution for $\phi$ at the grid point $(i, j)$, and $\phi_{i, j}^{\text {old }}$ denotes the current old value for $\phi$ at the same grid point. Under the framework of time-marching approach, the approximations of derivatives $\phi_{x}^{-}, \phi_{x}^{+}, \phi_{y}^{-}, \phi_{y}^{+}$in (3.4) are calculated from the old values of $\phi$ at grid points. First order upwind finite difference or high order WENO approximations ([23, 14, 39]) can be employed to calculate those derivatives. Due to the explicit framework of (3.4), it does not involve the inverse operations of the nonlinear Hamiltonian $H$ and the nonlinear equations as (2.1), so it can solve general Hamilton-Jacobi equations using any monotone numerical Hamiltonian and high order nonlinear approximations easily. However, the time-marching approach to the steady state calculation is constrained by the CFL condition and a finite speed of propagation so the number of iterations needed is at least proportional to the number of grids in each dimension.

In order to keep the simple explicit framework of the fixed-point iteration (3.4) and accelerate its convergence speed, we adopt the Gauss-Seidel idea and alternating sweeping strategy to the fixed-point iteration (3.4) in the following way. When the first order upwind approximations or high order WENO approximations ([23, 14, 39]) for derivatives $\left(\phi_{x}\right)_{i, j}^{-},\left(\phi_{x}\right)_{i, j}^{+},\left(\phi_{y}\right)_{i, j}^{-},\left(\phi_{y}\right)_{i, j}^{+}$in (3.4) are computed, we always use the newest available values for $\phi$ in the interpolation stencils according to the philosophy of Gauss-Seidel iterations. Of course, since we have not updated $\phi_{i, j}$ yet, $\phi_{i, j}^{\text {old }}$ is used in calculations of $\left(\phi_{x}\right)_{i, j}^{-},\left(\phi_{x}\right)_{i, j}^{+},\left(\phi_{y}\right)_{i, j}^{-},\left(\phi_{y}\right)_{i, j}^{+}$. Furthermore, the iterations do not just proceed in only one direction $i=1: I, j=1: J$ as the time-marching approach, but in the following four alternating directions repeatedly,

$$
\begin{aligned}
& \text { (1) } i=1: I, j=1: J \\
& \text { (2) } i=I: 1, j=1: J \\
& \text { (3) } i=I: 1, j=J: 1 ; \\
& \text { (4) } i=1: I, j=J: 1 .
\end{aligned}
$$

Since the strategy of alternating direction sweepings utilizes the characteristics property of hyperbolic PDEs, combining with the Gauss-Seidel philosophy, we will observe the acceleration of convergence speed for time-marching approach, which will be shown in the following numerical experiments. However, since this is not a true Gauss-Seidel algorithm we will not have a finite number of iterations independent of mesh size for convergence in general. The Godunov and Lax-Friedrichs numerical Hamiltonians are chosen to be the representatives of typical monotone numerical Hamiltonians. Godunov numerical Hamiltonian is pure upwind, and Lax-Friedrichs numerical Hamiltonian is not pure upwind, hence it has bigger numerical viscosity 
than Godunov numerical Hamiltonian. On the other hand, Lax-Friedrichs numerical Hamiltonian is simpler and easier to implement than the Godunov numerical Hamiltonian. A complete list and detailed discussion of monotone numerical Hamiltonians can be found in [33].

Basically, in the following numerical experiments we will compare the convergence behavior of the original time-marching type fixed-point iteration and the one with Gauss-Seidel philosophy and alternating direction sweepings. When we apply high order approximations to derivatives, we always use the third order WENO approximations [14]. On the boundary of computational domain, the extrapolation boundary conditions are applied [39]. For the simplicity of expression, from now on the original time-marching type fixed-point iteration (3.4) will be called "the timemarching method", the fixed-point iteration (3.4) combining with the Gauss-Seidel philosophy and alternating sweepings will be called "GS with sweeping method", and the fixed-point iteration (3.4) combining with the Gauss-Seidel philosophy but without alternating sweepings will be called "GS w/o sweeping method" The typical problem we select to study is the classical shape-from-shading problem [29]:

shape-from-shading 1. Eikonal equation (2.5) with

$$
f(x, y)=2 \pi \sqrt{[\cos (2 \pi x) \sin (2 \pi y)]^{2}+[\sin (2 \pi x) \cos (2 \pi y)]^{2}} .
$$

$\Gamma=\left\{\left(\frac{1}{4}, \frac{1}{4}\right),\left(\frac{3}{4}, \frac{3}{4}\right),\left(\frac{1}{4}, \frac{3}{4}\right),\left(\frac{3}{4}, \frac{1}{4}\right),\left(\frac{1}{2}, \frac{1}{2}\right)\right\}$, consisting of five isolated points. The computational domain $\Omega=[0,1] \times[0,1] . \phi(x, y)=0$ is prescribed at the boundary of the unit square. The solution for shape-from-shading problem is the shape function, which has the brightness $I(x, y)=1 / \sqrt{1+f(x, y)^{2}}$ under vertical lighting. See [29] for details. If we specify

$$
g\left(\frac{1}{4}, \frac{1}{4}\right)=g\left(\frac{3}{4}, \frac{3}{4}\right)=g\left(\frac{1}{4}, \frac{3}{4}\right)=g\left(\frac{3}{4}, \frac{1}{4}\right)=1, g\left(\frac{1}{2}, \frac{1}{2}\right)=2,
$$

then the exact solution for this case is

$$
\phi(x, y)= \begin{cases}\max (|\sin (2 \pi x) \sin (2 \pi y)|, 1+\cos (2 \pi x) \cos (2 \pi y)), & \text { if }|x+y-1|<\frac{1}{2} \text { and }|x-y|<\frac{1}{2} \\ |\sin (2 \pi x) \sin (2 \pi y)|, & \text { otherwise; }\end{cases}
$$

this solution is not smooth. In [14], high order time marching WENO schemes are used to calculate the solution for this problem.

3.1. Godunov numerical Hamiltonian. In this subsection, we use the Godunov numerical Hamiltonian. Godunov numerical Hamiltonian for the general Hamiltonian $H(u, v)$ has the form

$$
\hat{H}^{G}\left(u^{-}, u^{+} ; v^{-}, v^{+}\right)=e x t_{u \in I\left(u^{-}, u^{+}\right)} e x t_{v \in I\left(v^{-}, v^{+}\right)} H(u, v),
$$

where

$$
I(a, b)=[\min (a, b), \max (a, b)]
$$

and the function ext is defined by

$$
\operatorname{ext}_{u \in I(a, b)}= \begin{cases}\min _{a \leq u \leq b} & \text { if } a \leq b \\ \max _{b \leq u \leq a} & \text { if } a>b\end{cases}
$$



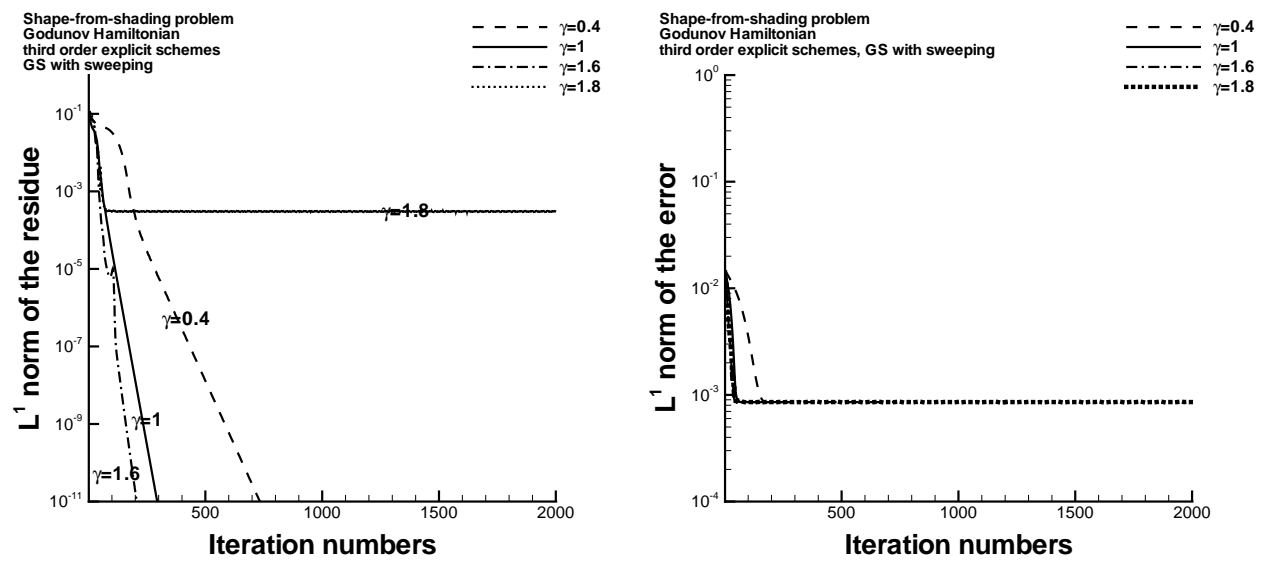

FIG. 3.1. Study on different $\gamma$ 's. Shape-from-shading 1, Godunov numerical Hamiltonian, the third order GS with sweeping method on $80 \times 80$ mesh. $\gamma=0.4,1,1.6,1.8$. Left: residue ${ }^{(n)}$; right: error $e^{(n)}$.

For the Eikonal equation case, it is simplified to

$$
\hat{H}^{G}\left(u^{-}, u^{+} ; v^{-}, v^{+}\right)=\sqrt{\max \left\{\left(u^{-}\right)^{+},\left(u^{+}\right)^{-}\right\}^{2}+\max \left\{\left(v^{-}\right)^{+},\left(v^{+}\right)^{-}\right\}^{2}},
$$

where $x^{+}=\max (x, 0), x^{-}=-\min (x, 0)$.

Now, we first use the third order GS with sweeping method to solve the shapefrom-shading problem 1 (3.5). The initial guess is the solution from the first order Godunov fast sweeping method [40] reviewed in Section 2. Numerical study on the parameter $\gamma$ in (3.4) shows that if $\gamma \leq 1.6$, the third order GS with sweeping method can converge for this problem. Figure (3.1) shows the residue $r^{(n)}(2.2)$ and the error $e^{(n)}$ (2.4) for the third order GS with sweeping method with different $\gamma$ on $80 \times 80$ mesh. From Figure (3.1) we can see that if $\gamma \leq 1.6$, the method converges and the nonlinear residue $r^{(n)}$ decreases to be less than $10^{-11}$. And with bigger $\gamma$, the method converges faster. But when $\gamma$ is increased to 1.8, the method fails to converge in 2000 iterations. The methods with different $\gamma$ 's generate same numerical error once they converge. An interesting observation is that at this grid size, even though the method with $\gamma=1.8$ fails to converge, it has the same numerical error as other $\gamma$ 's since the truncation error from the discretization of the PDE on this grid is more dominant than the error introduced by the final residue. In terms of convergence speed, $\gamma=1.6$ is optimal for the third order GS with sweeping method on the $80 \times 80$ mesh for this problem. Next we fix $\gamma=1.6$ and do the mesh refinement study for the third order GS with sweeping method. The results are plotted in Figure (3.2), and we can see that from the coarsest mesh $80 \times 80$ to the finest mesh $640 \times 640$, the iteration number for the method to converge is only increased from 180 to 250 , which shows that the computational complexity is much better than $O\left(N^{3 / 2}\right)$, which is the complexity for the time-marching method. And after the method converges, the second order $L^{1}$ accuracy is obtained if the error is measured on the whole region, which is reasonable because the exact solution of this problem is not smooth [14, 39].

Next we compare the time-marching method, the GS with sweeping method and the GS w/o sweeping method, for different $\gamma$ 's on the $80 \times 80$ mesh. We still use the third order WENO approximations for derivatives. Results of convergence history are plotted in Figure (3.3). In this case, the time-marching method can only converge 

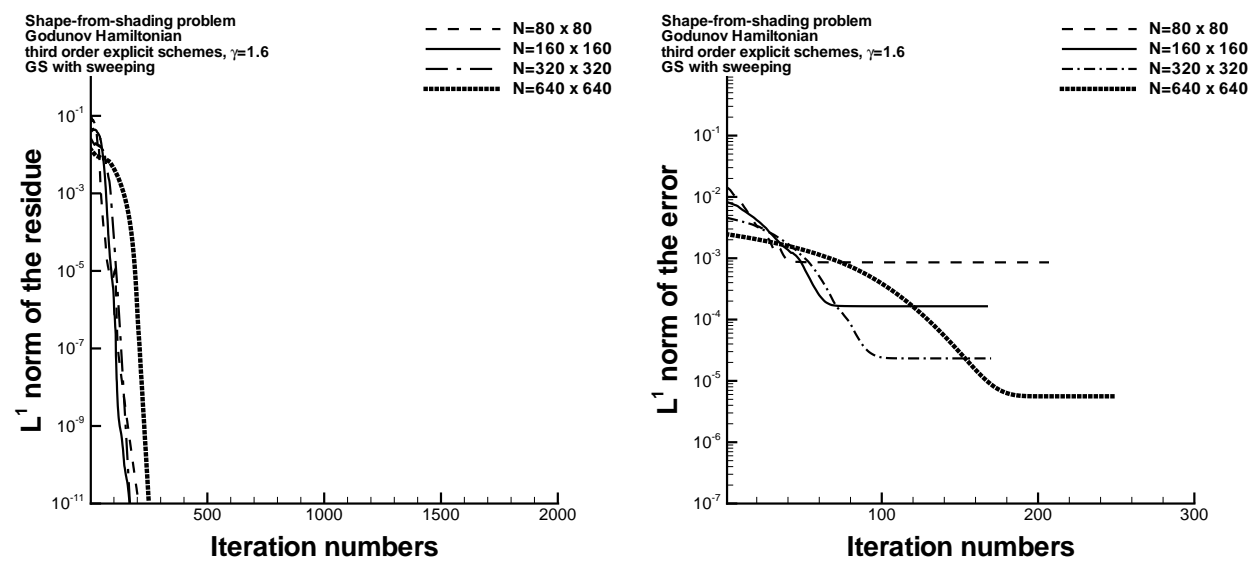

FIG. 3.2. Mesh refinement study. Shape-from-shading 1, Godunov numerical Hamiltonian, the third order GS with sweeping method with $\gamma=1.6$. Left: residue $r^{(n)}$; right: error $e^{(n)}$.

when $\gamma$ is as small as 0.4. It fails to converge at bigger $\gamma$ and blows up if $\gamma$ is increased to 1.6. Both GS with sweeping and GS w/o sweeping can converge at a much bigger $\gamma$, although GS with sweeping can converge at a slightly bigger $\gamma$ than GS w/o sweeping (GS with sweeping fails to converge at $\gamma=1.8$ from Figure (3.1), while GS w/o sweeping fails to converge at $\gamma=1.6$ ). A bigger $\gamma$ can lead to smaller iteration numbers if the method does converge under this $\gamma$. When $\gamma=0.4$, all of three methods converge, but both GS with sweeping and w/o sweeping converge three times faster than the time-marching method. And when $\gamma=1.6$, GS with sweeping method can converge in 200 iterations, but the other two methods diverges. The comparison here shows that the GS with sweeping method converges to steady states three times faster than time-marching approach under the same $\gamma$, if the time-marching method converges at this $\gamma$. And another advantage of the GS with sweeping method is that it has the biggest $\gamma$ among those three methods. The $\gamma$ actually represents the CFL number in the time-marching framework, and bigger $\gamma$ can make the method converge faster if the method does converge under this $\gamma$.

So far the initial guess we used is the solution from the first order Godunov fast sweeping method. In [14], the time evolution is initialized by function

$$
\phi(x, y, 0)=4 \min (\min (x, 1-x), \min (y, 1-y)) .
$$

Next, we use this initial guess in the GS with sweeping method and investigate its convergence history. The picture of $L^{1}$ norm of the residue $r^{(n)}(2.2)$ vs. iteration numbers is plotted in Figure (3.4) for successively refined meshes. The convergence pattern with initial guess (3.10) is similar to that with initial guess from first order fast sweeping solution, although more iterations are needed on the $N=640 \times 640$ mesh for the initial guess (3.10). We also plot the three-dimensional pictures of the initial function and the convergent numerical solution in Figure (3.5).

In the previous discussion, we only considered the time-marching method which is evolved by the forward Euler scheme. More often, the TVD Runge-Kutta schemes $[35,34]$ are used to evolve the high order schemes for HJ equations (1.2). Next we will show that the Gauss-Seidel philosophy and alternating direction sweepings can also be applied to Runge-Kutta schemes to accelerate the convergence to steady states. We use the second order TVD Runge-Kutta scheme [35, 34] as an example, then the 


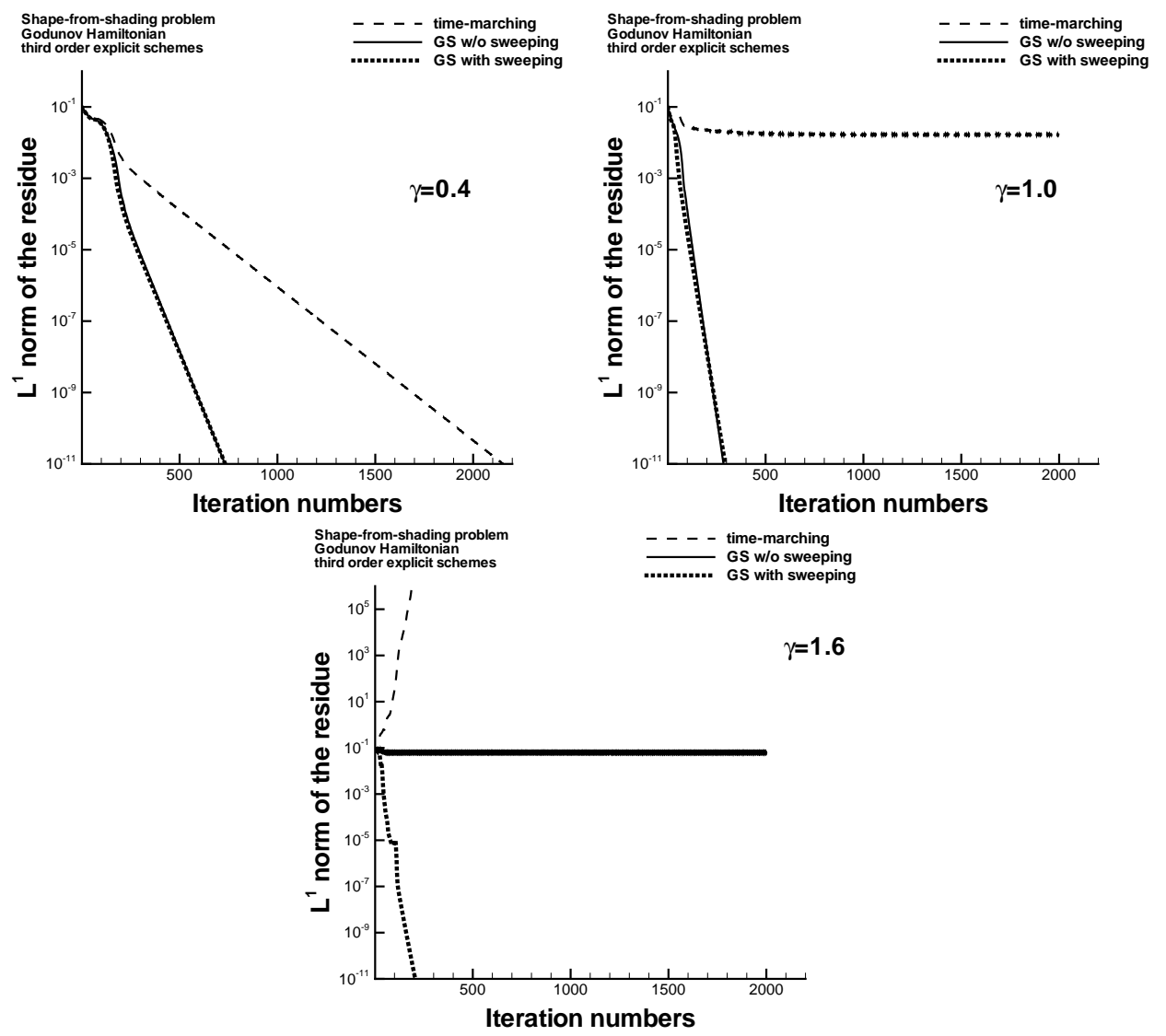

FIG. 3.3. Comparison between the third order time marching, GS w/o sweeping and GS with sweeping methods. Shape-from-shading 1, Godunov numerical Hamiltonian. $80 \times 80$ mesh. Top left: $\gamma=0.4$; top right: $\gamma=1$; bottom: $\gamma=1.6$.

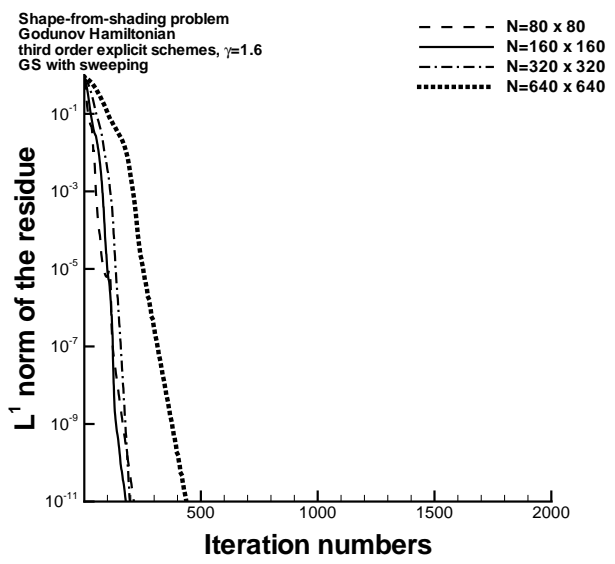

FIG. 3.4. Residue $r^{(n)}$ vs. iteration numbers. Mesh refinement study. Shape-from-shading 1, Godunov numerical Hamiltonian, the third order GS with sweeping method with $\gamma=1.6$, using the initial guess (3.10). 

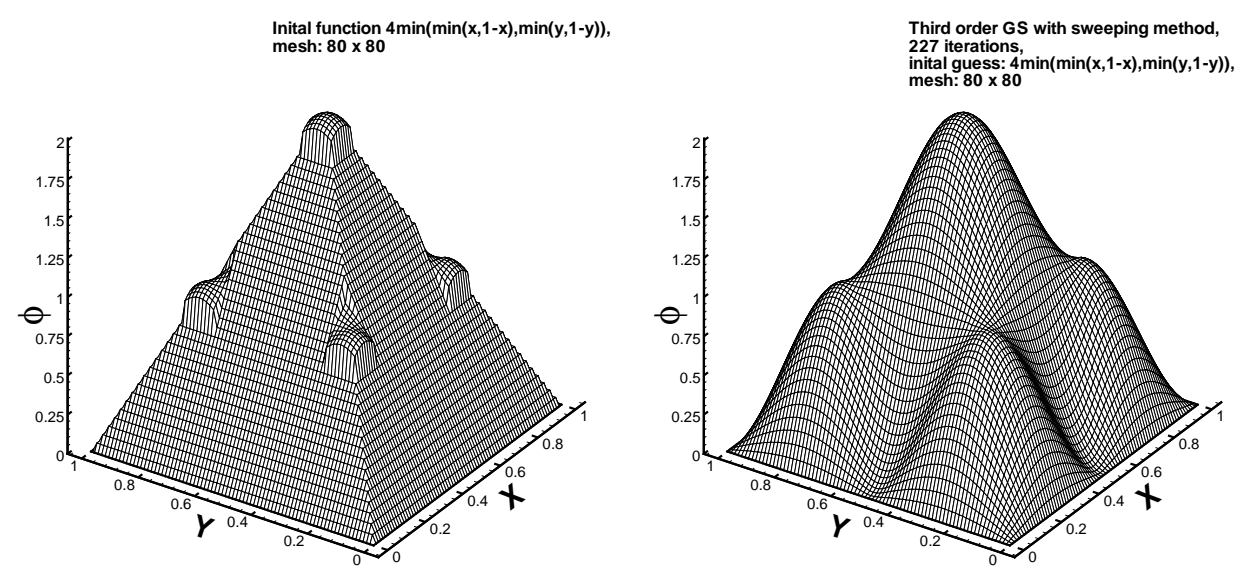

FIG. 3.5. shape-from-shading 1, Godunov numerical Hamiltonian, the third order GS with sweeping method with $\gamma=1.6$, using the initial guess (3.10). Left: three-dimensional view of the initial function (3.10); right: three-dimensional view of the solution after convergence in 227 iterations.

time-marching method is

$$
\begin{aligned}
\phi_{i, j}^{(1)} & =\phi_{i, j}^{n}+\Delta t\left[f_{i, j}-\hat{H}\left(\left(\phi_{x}\right)_{i, j}^{n,-},\left(\phi_{x}\right)_{i, j}^{n,+} ;\left(\phi_{y}\right)_{i, j}^{n,-},\left(\phi_{y}\right)_{i, j}^{n,+}\right)\right] \\
\phi_{i, j}^{n+1} & =\frac{1}{2} \phi_{i, j}^{n}+\frac{1}{2} \phi_{i, j}^{(1)}+\frac{1}{2} \Delta t\left[f_{i, j}-\hat{H}\left(\left(\phi_{x}\right)_{i, j}^{(1),-},\left(\phi_{x}\right)_{i, j}^{(1),+} ;\left(\phi_{y}\right)_{i, j}^{(1),-},\left(\phi_{y}\right)_{i, j}^{(1),+}\right)\right] .
\end{aligned}
$$

Applying the Gauss-Seidel philosophy, we obtain the following fixed-point iterative method, written in the pseudo-code form:

do $i=1: I, j=1: J$,

$$
\begin{aligned}
& \phi_{i, j}^{(1)}=\phi_{i, j}^{n}+\gamma\left(\frac{1}{\frac{\alpha_{x}}{h_{x}}+\frac{\alpha_{y}}{h_{y}}}\right)\left[f_{i, j}-\hat{H}\left(\left(\phi_{x}\right)_{i, j}^{-},\left(\phi_{x}\right)_{i, j}^{+} ;\left(\phi_{y}\right)_{i, j}^{-},\left(\phi_{y}\right)_{i, j}^{+}\right)\right] \\
& \quad \text { enddo. } \\
& \quad \text { do } i=1: I, j=1: J \\
& \phi_{i, j}^{n+1}=\phi_{i, j}^{(1)}+\frac{1}{2} \gamma\left(\frac{1}{\frac{\alpha_{x}}{h_{x}}+\frac{\alpha_{y}}{h_{y}}}\right)\left[f_{i, j}-\hat{H}\left(\left(\phi_{x}\right)_{i, j}^{-},\left(\phi_{x}\right)_{i, j}^{+} ;\left(\phi_{y}\right)_{i, j}^{-},\left(\phi_{y}\right)_{i, j}^{+}\right)\right]
\end{aligned}
$$

enddo.

Again, we always use the newest available values of $\phi$ in the interpolation stencils to compute the approximations for derivatives $\left(\phi_{x}\right)_{i, j}^{-},\left(\phi_{x}\right)_{i, j}^{+},\left(\phi_{y}\right)_{i, j}^{-},\left(\phi_{y}\right)_{i, j}^{+}$in $(3.12)$ and (3.13). From the point of view of implementation, we just use one array for all of these $\phi^{n}, \phi^{(1)}$ and $\phi^{n+1}$. Also four alternating direction sweepings

$$
\begin{aligned}
& \text { (1) } i=1: I, j=1: J ; \\
& \text { (2) } i=I: 1, j=1: J ; \\
& \text { (3) } i=I: 1, j=J: 1 ;
\end{aligned}
$$



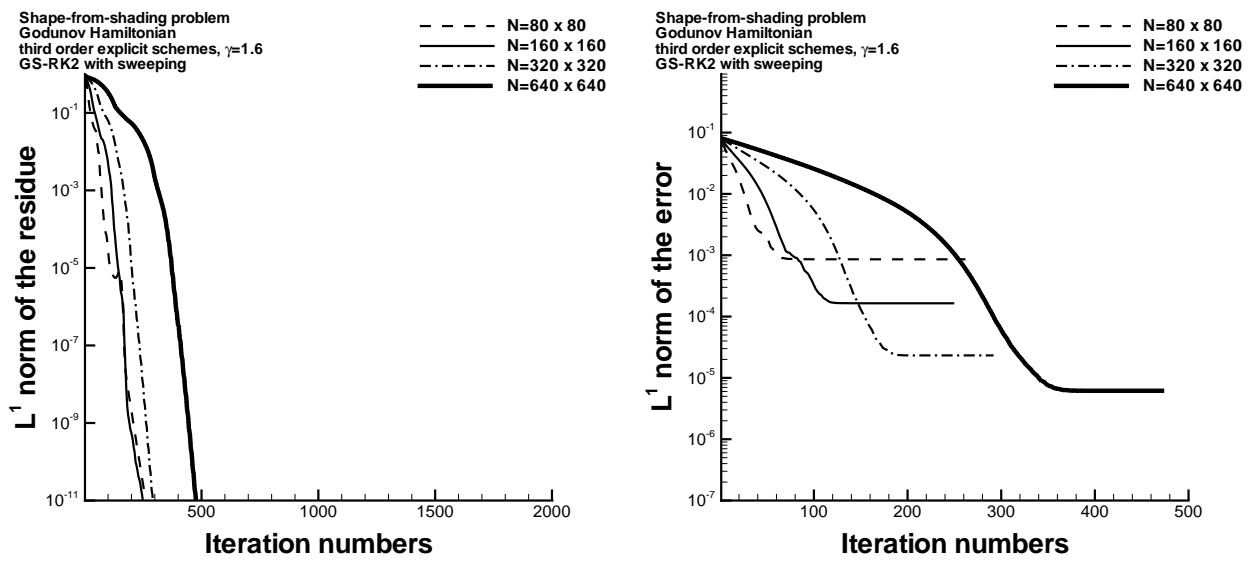

Fig. 3.6. Mesh refinement study. Shape-from-shading 1, Godunov numerical Hamiltonian, Third order GS-RK2 sweeping method with $\gamma=1.6$, using the initial guess (3.10). Left: residue $r^{(n)}$; right: error $e^{(n)}$.

$$
\text { (4) } i=1: I, j=J: 1 \text {. }
$$

are applied repeatedly for both (3.12) and (3.13), and note that the sweeping direction of (3.12) and (3.13) should be same during one sweeping. Now since one sweeping includes both iterations of $\phi^{(1)}$ and $\phi^{n+1}$, it should be counted as 2 iterations when we count the iteration numbers. We name this method as "GS-RK2 sweeping method" in this paper. We applied this method with $\gamma=1.6$ to the shape-from-shading problem 1, using Godunov numerical Hamiltonian, third order WENO approximations for derivatives and the initial guess (3.10). The convergence history and accuracy are plotted in Figure (3.6). Basically the convergence history is similar as the forward Euler cases in Figure (3.4), and the second order $L^{1}$ accuracy is obtained if the error is measured on the whole region, due to the non-smoothness of the exact solution of this problem.

Next we compare the convergence history of GS-RK2 sweeping method and the original time-marching method using TVD-RK2 (3.11). We plot the picture of residue vs. iteration numbers for both methods on the $320 \times 320$ and $640 \times 640$ meshes in Figure (3.7). This Figure shows that the GS-RK2 sweeping method converges to steady states twice faster than the time marching RK2 method.

To make the comparison complete, we also use the first order upwind approximations for derivatives in the GS sweeping method and in the time-marching method $(3.4,3.1)$. The convergence history for both of the methods on the $320 \times 320$ and $640 \times 640$ meshes is plotted in Figure (3.8). From the Figure, we see that the first order GS sweeping method converges to steady states more than 1.6 times faster than the first order time marching method.

3.2. Lax-Friedrichs numerical Hamiltonian. We use the Lax-Friedrichs numerical Hamiltonian in this subsection. The Lax-Friedrichs numerical Hamiltonian for a Hamiltonian $H(u, v)$ has the form

$$
\hat{H}^{L F}\left(u^{-}, u^{+} ; v^{-}, v^{+}\right)=H\left(\frac{u^{-}+u^{+}}{2}, \frac{v^{-}+v^{+}}{2}\right)-\frac{1}{2} \alpha_{x}\left(u^{+}-u^{-}\right)-\frac{1}{2} \alpha_{y}\left(v^{+}-v^{-}\right)
$$

where $\alpha_{x}$ and $\alpha_{y}$ are same as the definition in (3.3). 


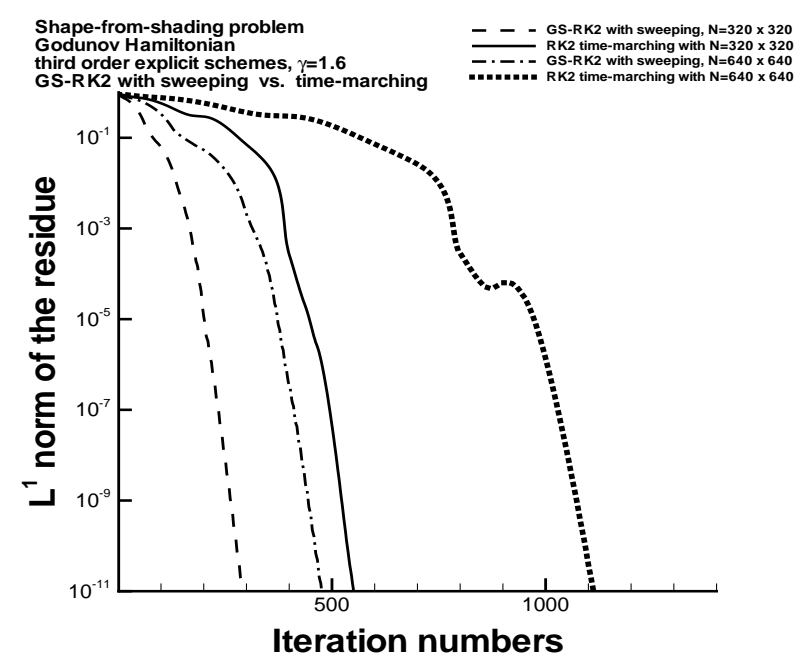

FIG. 3.7. Comparison between the third order GS-RK2 sweeping method and the time marching RK2 method (3.11) with $\gamma=1.6$. Shape-from-shading 1, Godunov numerical Hamiltonian, using the initial guess (3.10). Both $320 \times 320$ and $640 \times 640$ meshes. Residue $r^{(n)}$ vs. iteration numbers.

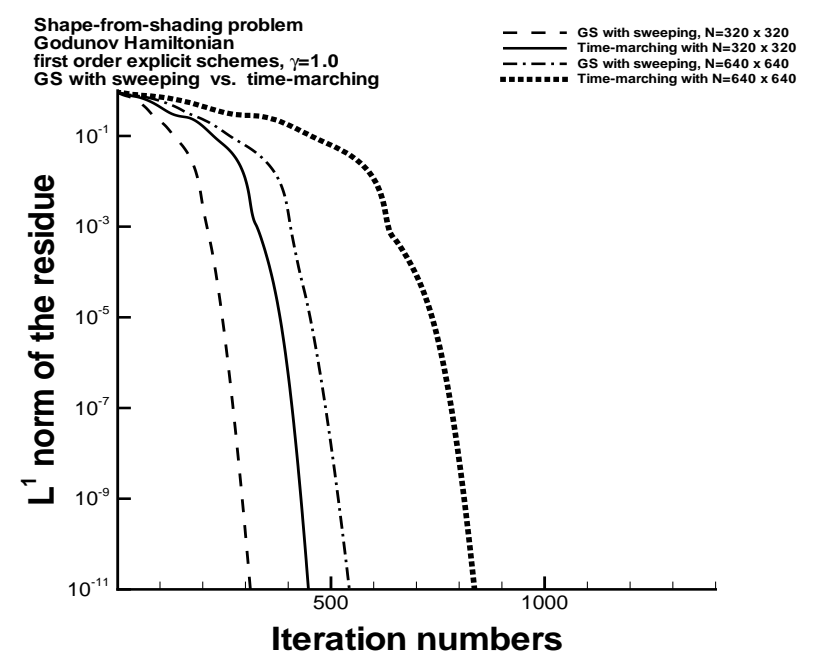

FIG. 3.8. Comparison of iteration numbers between the first order GS with sweeping and direct time-marching method. $\gamma=1.0$. Shape-from-shading 1, Godunov numerical Hamiltonian, using the initial guess (3.10). Both $320 \times 320$ and $640 \times 640$ meshes. Residue $r^{(n)}$ vs. iteration numbers.

Now, we first solve the shape-from-shading problem 1 (3.5) by using the third order GS with sweeping method, with the Lax-Friedrichs numerical Hamiltonian (3.14). The initial guess is the function (3.10) as in the previous subsection. Numerical study on the parameter $\gamma$ in (3.4) shows that if $\gamma \leq 1.3$, the third order GS with sweeping method can converge to the steady states. It fails to converge to the steady states when $\gamma$ increases to 1.4. We do the mesh refinement study for the third order GS with sweeping method for $\gamma=1.3$, and the results are plotted in Figure (3.9). From the Figure we can see that the pattern of convergence history is similar as that of the Godunov numerical Hamiltonian in Figure (3.2), from the coarsest mesh $80 \times 80$ 

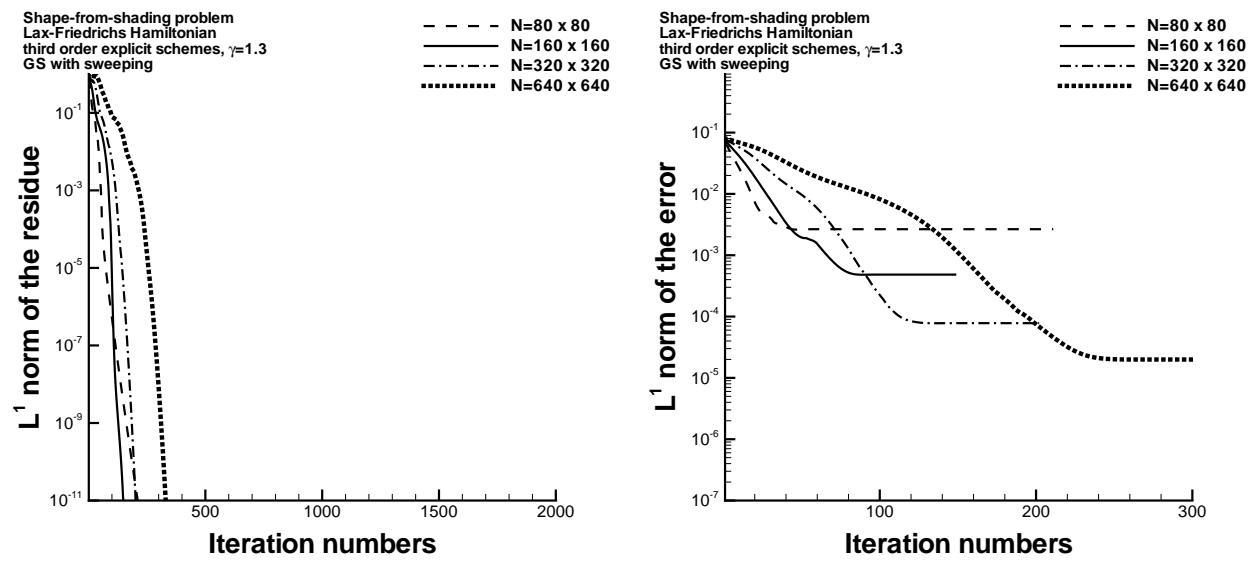

Fig. 3.9. Mesh refinement study. Shape-from-shading 1, Lax-Friedrichs numerical Hamiltonian, the third order GS with sweeping method with $\gamma=1.3$, using the initial guess (3.10). Left: residue $r^{(n)}$; right: error $e^{(n)}$.

to the refinest mesh $640 \times 640$, the iteration number for the method to converge to steady states is only increased from 150 to 330, which shows that the computational complexity is much better than $O\left(N^{3 / 2}\right)$. And after the method converges, the second order $L^{1}$ accuracy is obtained if the error is measured on the whole region due to the non-smoothness of the exact solution. Comparing the error values in Figure (3.9) and Figure (3.2), we see that the numerical error by the Lax-Friedrichs numerical Hamiltonian is bigger than that by the Godunov numerical Hamiltonian, which is reasonable because the Lax-Friedrichs numerical Hamiltonian has bigger numerical viscosity.

Again we compare the time-marching method, the GS with sweeping method and the GS w/o sweeping method, for different $\gamma$ 's on the $80 \times 80$ mesh. Results of convergence history are plotted in Figure (3.10). We see that the pattern is similar to that of the Godunov numerical Hamiltonian in Figure (3.3). Again, the timemarching method can only converge when $\gamma$ is as small as 0.4. It fails to converge at bigger $\gamma$ and blows up if $\gamma$ is increased to 1.3. Both GS with sweeping and GS w/o sweeping can converge at a much bigger $\gamma$ till $\gamma=1.3$. A bigger $\gamma$ can lead to smaller iteration numbers if the method does converge under this $\gamma$. When $\gamma=0.4$, all of the three methods converge, but both GS with sweeping and w/o sweeping converge three times faster than the time-marching method. And when $\gamma=1.3$, GS with sweeping method can converge in 200 iterations, GS w/o sweeping method can converge in 300 iterations, but the time-marching method blows up. The comparison here shows that the GS with sweeping method has the fastest convergence speed to steady states among these three methods and converges three times faster than the time-marching approach under the same $\gamma$, if the time-marching method does converge under this $\gamma$. The acceleration is even more when the grid is further refined. Another advantage of the GS with sweeping method is that it has much bigger $\gamma$ to guarantee convergence than the time-marching method. The $\gamma$ actually represents the CFL number in the time-marching framework, and bigger $\gamma$ can make the method converge to steady states faster if the method does converge under this $\gamma$.

Studies so far show that the fixed-point iterative sweeping methods with either the Godunov numerical Hamiltonian or the Lax-Friedrichs numerical Hamiltonian have 


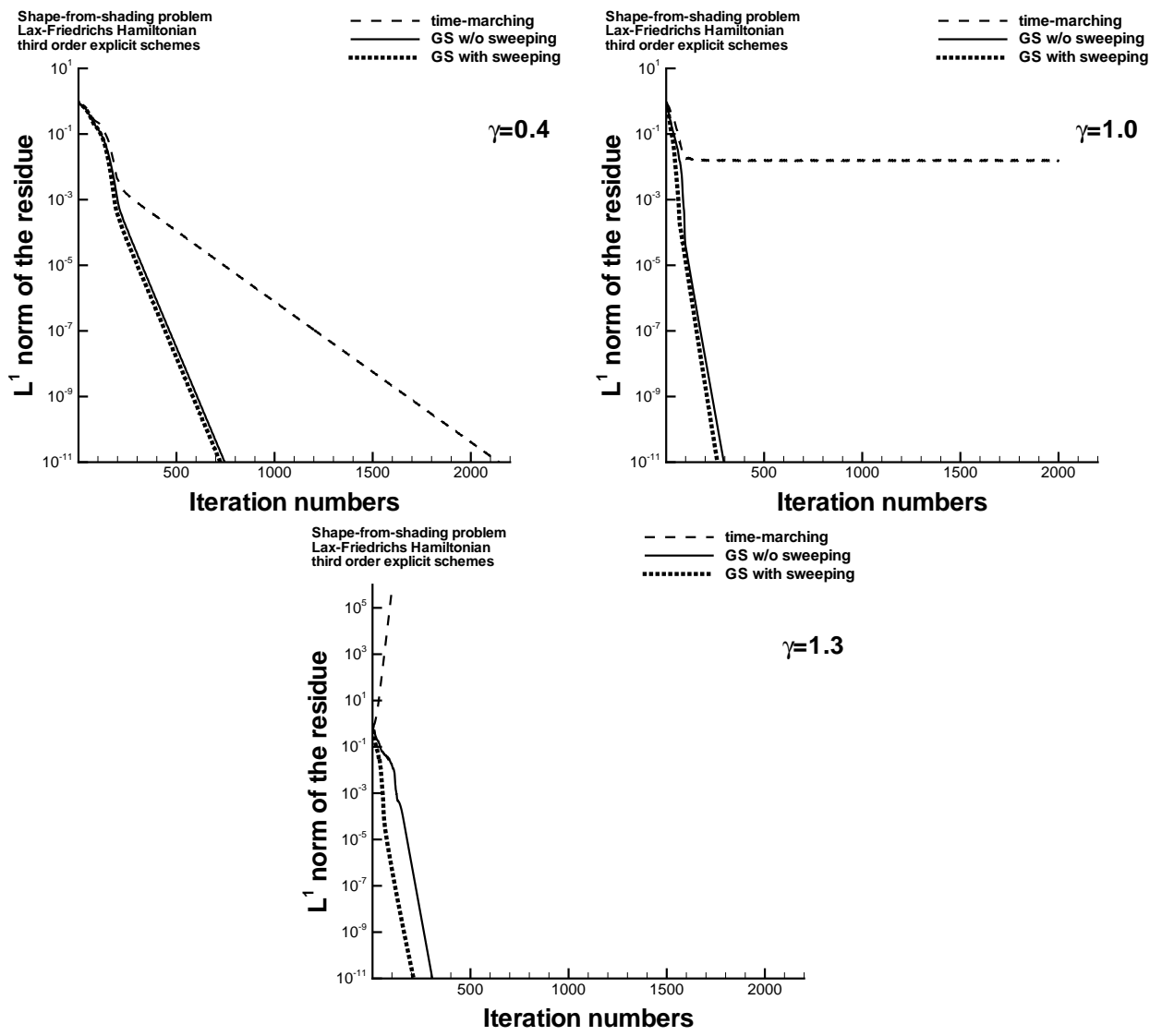

FIG. 3.10. Comparison between the third order time marching, Gauss-Seidel w/o sweeping and Gauss-Seidel with sweeping methods. Shape-from-shading 1, Lax-Friedrichs numerical Hamiltonian, using the initial guess (3.10), $80 \times 80$ mesh. Top left: $\gamma=0.4$; top right: $\gamma=1$; bottom: $\gamma=1.3$.

similar convergence pattern. But we want to show that although the Godunov numerical Hamiltonian is more complicated than the Lax-Friedrichs numerical Hamiltonian, it is pure upwind and has smaller numerical viscosity. We applied the third order GS sweeping method with both of numerical Hamiltonians to another shape-from-shading problem [29, 13]:

shape-from-shading 2. Eikonal equation (2.5) with

$$
f(x, y)=\sqrt{(1-|x|)^{2}+(1-|y|)^{2}} .
$$

The computational domain $\Omega=[-1,1] \times[-1,1] . \phi(x, y)=0$ is prescribed at the boundary of the square for both cases. The exact solution for the problem is

$$
\phi(x, y)=(1-|x|)(1-|y|) .
$$

The numerical results by the third order GS sweeping method with these two numerical Hamiltonians are plotted in Figure (3.11). We use the initial guess $\phi=0$ as in [13]. From Figure (3.11), we can see that the result by Godunov numerical Hamiltonian has a much better resolution on shocks than that by Lax-Friedrichs numerical Hamiltonian. Actually, in the $80 \times 80$ mesh, the $L^{1}$ error is $4.39 \times 10^{-8}$ and the 

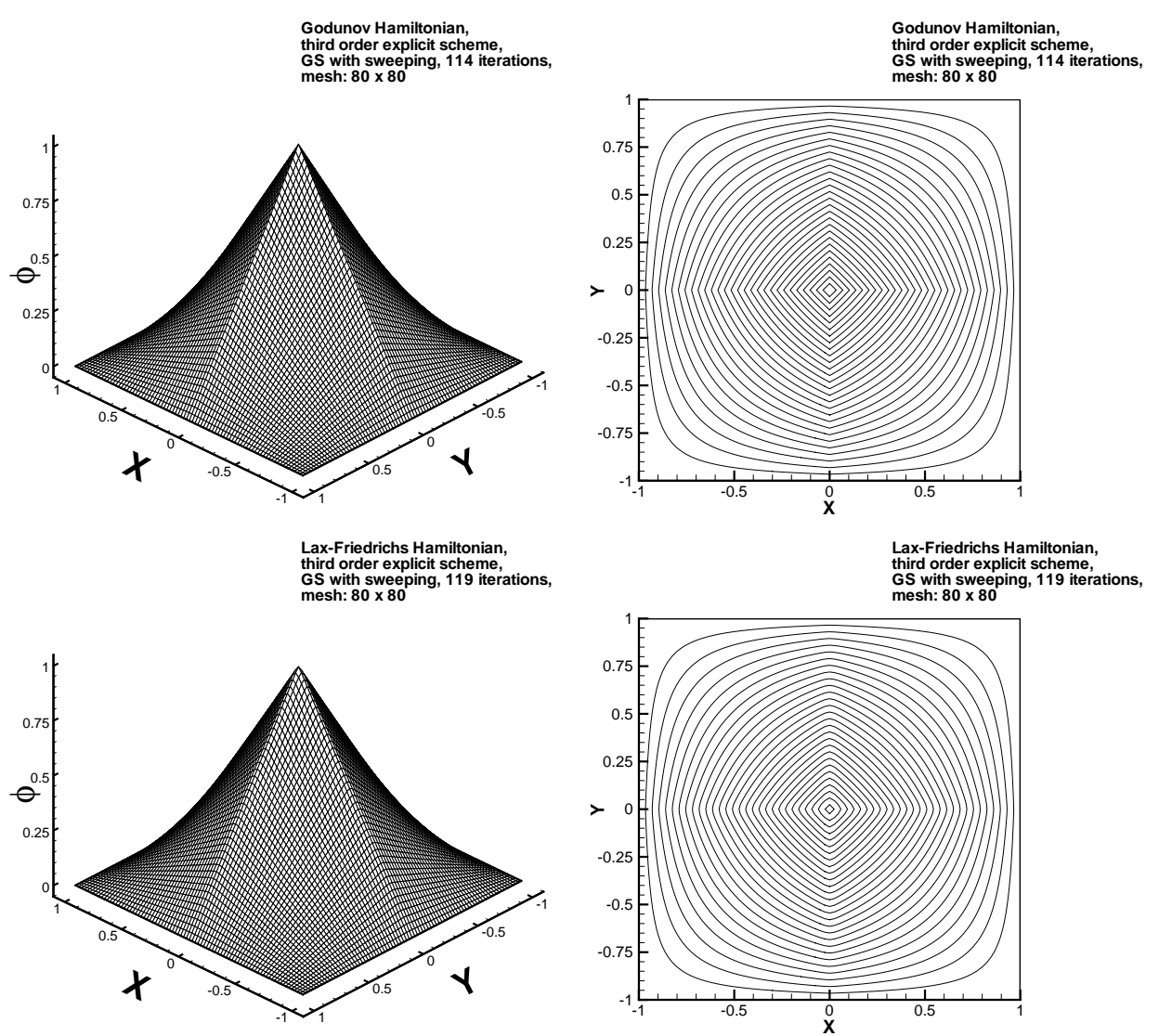

FIG. 3.11. Shape-from-shading 2. Resolution comparison between Godunov and Lax-Friedrichs numerical Hamiltonian. Third order GS with sweeping method by Godunov numerical Hamiltonian $(\gamma=1.6)$ and Lax-Friedrichs numerical Hamiltonian $(\gamma=1.0)$, using the initial guess $\phi=0$. $80 \times 80$ mesh. Top left: three-dimensional view of the result by Godunov numerical Hamiltonian; bottom left: three-dimensional view of the result by Lax-Friedrichs numerical Hamiltonian; top right: contour lines view of the result by Godunov numerical Hamiltonian, 30 equally spaced contour lines from $\phi=0$ to $\phi=1$; bottom right: contour lines view of the result by Lax-Friedrichs numerical Hamiltonian, 30 equally spaced contour lines from $\phi=0$ to $\phi=1$.

$L^{\infty}$ error is $6.89 \times 10^{-6}$ for Godunov, while the $L^{1}$ error is $1.93 \times 10^{-4}$ and the $L^{\infty}$ error is $1.53 \times 10^{-2}$ for Lax-Friedrichs. The optimal $\gamma$ is 1.6 for Godunov numerical Hamiltonian and 1.0 for Lax-Friedrichs numerical Hamiltonian in this example. The iteration numbers needed to reach the $L^{1}$ residue error $10^{-11}$ are 114 for Godunov and 119 for Lax-Friedrichs numerical Hamiltonian. So Godunov and Lax-Friedrichs numerical Hamiltonian are comparable in terms of iteration number, but the Godunov has a much sharper resolution than Lax-Friedrichs numerical Hamiltonian.

At last, we test the acceleration of convergence using a more complicated example in elastic wave propagation, which is an anisotropic Eikonal equation:

Travel-time problem. The quasi-P slowness surfaces are defined by the quadratic equation [25]:

$$
c_{1} \phi_{x}^{4}+c_{2} \phi_{x}^{2} \phi_{y}^{2}+c_{3} \phi_{y}^{4}+c_{4} \phi_{x}^{2}+c_{5} \phi_{y}^{2}+1=0
$$



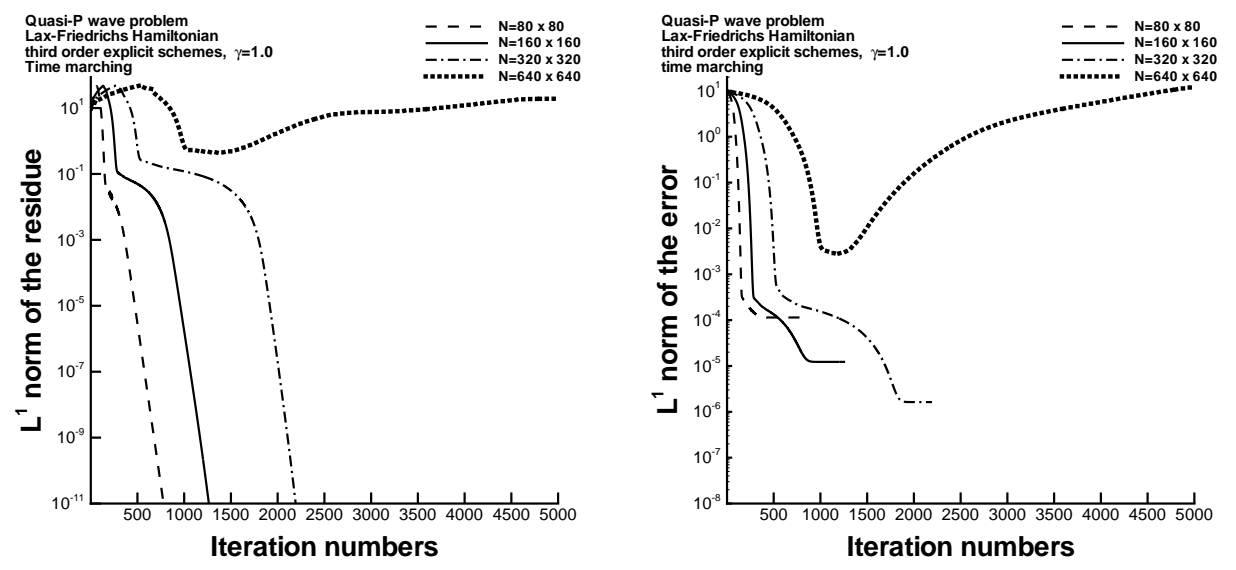

Fig. 3.12. Mesh refinement study. Travel-time problem, Lax-Friedrichs numerical Hamiltonian, the third order time-marching method with $\gamma=1.0$, using the initial guess 10 on the computational grid points. Left: residue $r^{(n)}$; right: error $e^{(n)}$.

where

$$
\begin{gathered}
c_{1}=a_{11} a_{44}, c_{2}=a_{11} a_{33}+a_{44}^{2}-\left(a_{13}+a_{44}\right)^{2}, \\
c_{3}=a_{33} a_{44}, c_{4}=-\left(a_{11}+a_{44}\right), c_{5}=-\left(a_{33}+a_{44}\right) .
\end{gathered}
$$

Here $a_{i j}$ s are given elastic parameters. The corresponding quasi-P wave eikonal equation is

$$
\sqrt{-\frac{1}{2}\left(c_{4} \phi_{x}^{2}+c_{5} \phi_{y}^{2}\right)+\sqrt{\frac{1}{4}\left(c_{4} \phi_{x}^{2}+c_{5} \phi_{y}^{2}\right)^{2}-\left(c_{1} \phi_{x}^{4}+c_{2} \phi_{x}^{2} \phi_{y}^{2}+c_{3} \phi_{y}^{4}\right)}}=1 .
$$

The elastic parameters are taken to be

$$
a_{11}=15.0638, a_{33}=10.8373, a_{13}=1.6381, a_{44}=3.1258
$$

in the numerical example to be shown. The computational domain is $[-1,1] \times[-1,1]$, and $\Gamma=\{(0,0)\}$. The Hamiltonians are pretty complicated, so we apply the LaxFriedrichs numerical Hamiltonian (3.14) to this problem. The initial guess is big values (we take 10) on the computational grid points, and $\alpha_{x}=\alpha_{y}=4, \gamma=1$.

First we use the time-marching approach, and the convergence history is plotted in Figure (3.12). We can see that more than 2000 iterations are needed for $320 \times 320$ mesh, and it even fails to converge for the $640 \times 640$ mesh. Then we apply the GS with sweeping and we can see that the convergence is significantly accelerated, in Figure (3.13). In Table (3.1), we list the iteration numbers for both methods on this case, and we can see a 5 times acceleration due to the usage of GS with sweeping, if the time-marching approach converges, and on $640 \times 640$ mesh, time-marching fails to converge but GS with sweeping can still converge. The three-dimensional and contour views of the numerical solution by GS with sweeping on $80 \times 80$ mesh are presented in Figure (3.14).

Remark 1. We want to emphasize that the local solver to which we apply the Gauss-Seidel philosophy and alternating sweeping strategy is the fixed-point iterative 

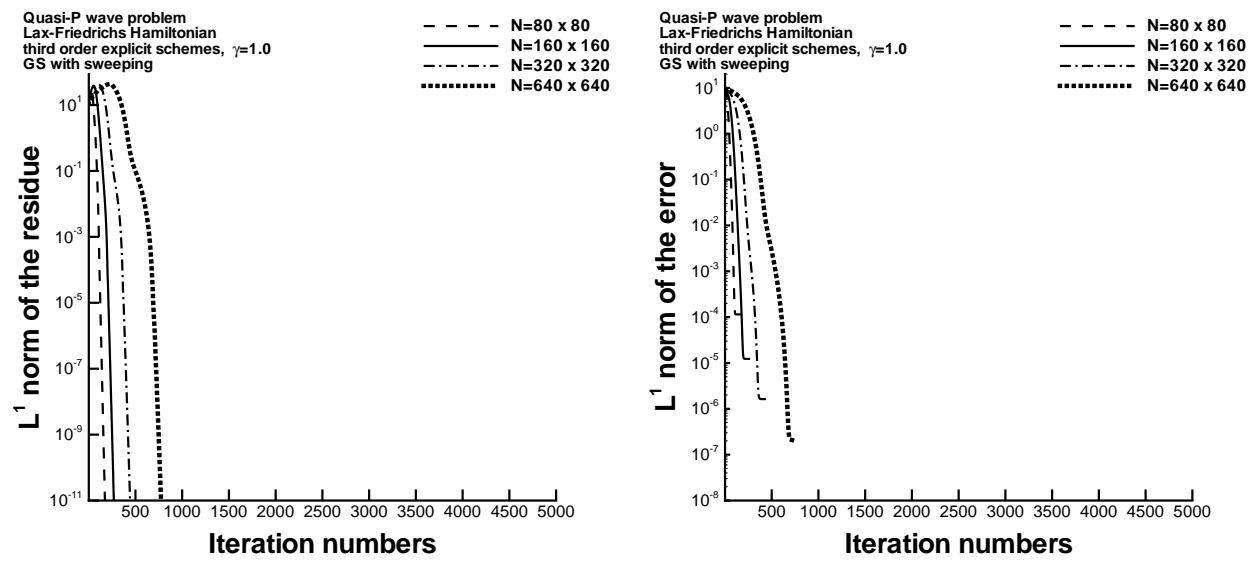

Fig. 3.13. Mesh refinement study. Travel-time problem, Lax-Friedrichs numerical Hamiltonian, the third order GS with sweeping method with $\gamma=1.0$, using the initial guess 10 on the computational grid points. Left: residue $r^{(n)}$; right: error $e^{(n)}$.

TABLE 3.1

Travel-time problem. Comparison of iteration numbers between time-marching and GS with sweeping methods. Lax-Friedrichs numerical Hamiltonian. The third order method with $\gamma=1.0$.

\begin{tabular}{|r|r|r|r|}
\hline mesh & iter \# of GS with sweeping & iter \# of time-marching & acce. ratio \\
\hline $80 \times 80$ & 176 & 780 & 4.43 \\
\hline $160 \times 160$ & 269 & 1264 & 4.70 \\
\hline $320 \times 320$ & 443 & 2194 & 4.95 \\
\hline $640 \times 640$ & 774 & Not conv. & $\infty$ \\
\hline
\end{tabular}

method (3.4). This local solver is explicit and does not involve solving nonlinear equations. This is different from the fast sweeping methods in [18, 28, 36, 40], where the local solvers are implicit and nonlinear equations have to be solved. In [39], the high order approximations are applied to the building blocks whose framework are still implicit. As a result, the Godunov version of the schemes in [39] is different from the Godunov GS sweeping method in this paper. But interestingly, it is easy to show that the Lax-Friedrichs GS sweeping methods in this paper are equivalent to the Lax-Friedrichs methods in $[17,39]$, although they are derived from different starting points.

Remark 2. It is easy to see that the combination of the time-marching type fixed-point iteration (3.4) with the Gauss-Seidel philosophy and alternating sweeping strategy does not add any additional computational cost to the original time-marching method.

Remark 3. It is also straightforward to use the method in this paper to apply the Gauss-Seidel philosophy and alternating sweeping strategy to the time-marching method for the steady states calculation of hyperbolic conservation laws. We expect that the convergence to steady states of hyperbolic conservation laws can be accelerated and the further study constitutes our future work.

4. Conclusion. In this paper, we explicitly apply the Gauss-Seidel philosophy and alternating sweeping strategy to the time-marching method to accelerate its con- 

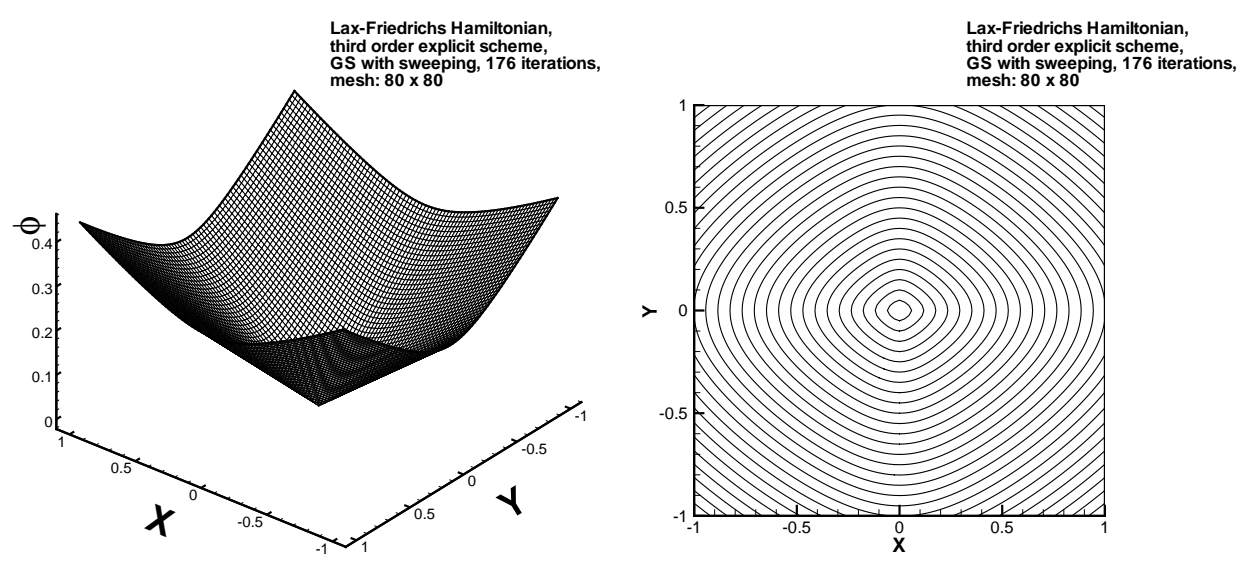

FIG. 3.14. Travel-time problem, Lax-Friedrichs numerical Hamiltonian, the third order GS with sweeping method with $\gamma=1.0$, using the initial guess 10 on the computational grid points. $80 \times 80$ mesh. Left: three-dimensional view; right: contour lines view, 30 equally spaced contour lines from $\phi=0$ to $\phi=0.44173$.

vergence to steady states. Extensive numerical experiments show both acceleration of convergence to steady states and a larger CFL number can be achieved for the original time-marching method, without any additional computational cost.

\section{REFERENCES}

[1] R. AbGRaLl, Numerical discretization of the first-order Hamilton-Jacobi equation on triangular meshes, Communications on Pure and Applied Mathematics, 49 (1996), pp. 1339-1373.

[2] S. Augoula and R. Abgrall, High order numerical discretization for Hamilton-Jacobi equations on triangular meshes, Journal of Scientific Computing, 15 (2000), pp. 197-229.

[3] M. BouÉ AND P. DuPUIS, Markov chain approximations for deterministic control problems with affine dynamics and quadratic cost in the control, SIAM Journal on Numerical Analysis, 36 (1999), pp. 667-695.

[4] S. BRYSON AND D. LEVY, High-order central WENO schemes for multidimensional HamiltonJacobi equations, SIAM Journal on Numerical Analysis, 41 (2003), pp. 1339-1369.

[5] T. Barth And J. Sethian, Numerical schemes for the Hamilton-Jacobi and level set equations on triangulated domains, Journal of Computational Physics, 145 (1998), pp. 1-40.

[6] T. Cecil, J. Qian and S. Osher, Numerical methods for high dimensional Hamilton-Jacobi equations using radial basis functions, Journal of Computational Physics, 196 (2004), pp. 327-347.

[7] M . G. Crandall and P. L. Lions, Viscosity solutions of Hamilton-Jacobi equations, Trans. Amer. Math. Soc., 277 (1983), pp. 1-42.

[8] J. Dellinger And W. W. Symes, Anisotropic finite-difference traveltimes using a HamiltonJacobi solver, 67th Ann. Internat. Mtg., Soc. Expl. Geophys., Expanded Abstracts, 1997, pp. $1786-1789$.

[9] M. Falcone and R. Ferretti, Discrete time high-order schemes for viscosity solutions of Hamilton-Jacobi-Bellman equations, Numer. Math., 67 (1994), pp. 315-344.

[10] M. Falcone And R. Ferretti, Semi-Lagrangian schemes for Hamilton-Jacobi equations, discrete representation formulae and Godunov methods, Journal of Computational Physics, 175 (2002), pp. 559-575.

[11] S. GRAY AND W. MAY, Kirchhoff migration using eikonal equation travel-times, Geophysics, 59 (1994), pp. 810-817.

[12] J. Helmsen, E. Puckett, P. Colella And M. DorR, Two new methods for simulating photolithography development in 3d, Proc. SPIE, 2726 (1996), pp. 253-261.

[13] C. Hu AND C.-W. Shu, A discontinuous Galerkin finite element method for Hamilton-Jacobi equations, SIAM Journal on Scientific Computing, 20 (1999), pp. 666-690. 
[14] G.-S. JiAng AND D. Peng, Weighted ENO Schemes for Hamilton-Jacobi equations, SIAM Journal on Scientific Computing, 21 (2000), pp. 2126-2143.

[15] S. JIN AND Z. XIN, Numerical passage from systems of conservation laws to HamiltonJacobi equations and relaxation schemes, SIAM Journal on Numerical Analysis, 35 (1998), pp. 2385-2404.

[16] S. KIM AND R. COOK, 3D traveltime computation using second-order ENO scheme, Geophys. 64 (1999), pp. $1867-1876$.

[17] C.Y. KaO, S. Osher AND J. QIAN, Lax-Friedrichs sweeping scheme for static Hamilton-Jacobi equations, Journal of Computational Physics, 196 (2004), pp. 367-391.

[18] C.Y. KaO, S. Osher And Y.H. Tsai, Fast Sweeping Methods for static Hamilton-Jacobi Equations, SIAM Journal on Numerical Analysis, 42 (2005), pp. 2612-2632.

[19] P.L. Lions, E. Rouy And A. Tourin, Shape-from-shading, viscosity solutions and edges, Numer. Math., 64 (1993), pp. 323-353.

[20] C.-T. LIN AND E. TADMOR, High-resolution non-oscillatory central schemes for approximate Hamilton-Jacobi equations, SIAM Journal on Scientific Computing, 21 (2000), pp. 21632186.

[21] S. OsHeR, A level set formulation for the solution of the Dirichlet problem for Hamilton-Jacobi equations, SIAM J. Math. Anal., 24 (1993), pp. 1145-1152.

[22] S. Osher AND J. Sethian, Fronts propagating with curvature dependent speed: algorithms based on Hamilton-Jacobi formulations, Journal of Computational Physics, 79 (1988), pp. 12-49.

[23] S. Osher AND C.-W. Shu, High-order essentially nonoscillatory schemes for Hamilton-Jacobi equations, SIAM Journal on Numerical Analysis, 28 (1991), pp. 907-922.

[24] D. Peng, S. Osher, B. Merriman, H.-K. Zhao and M. Rang, A PDE-based fast local level set method, Journal of Computational Physics, 155 (1999), pp. 410-438.

[25] J. Qian, L.T. Cheng And S. Osher, A level set based Eulerian approach for anisotropic wave propagations, Wave Motion, 37 (2003), pp. 365-379.

[26] J. QIan And W. W. Symes, Paraxial eikonal solvers for anisotropic quasi-P travel times, Journal of Computational Physics, 174 (2001), pp. 256-278.

[27] J. QIAN And W. W. Symes, Finite-difference quasi-P traveltimes for anisotropic media, Geophysics, 67 (2002), pp. 147-155.

[28] J. QIan, Y.-T. Zhang AND H.-K. ZhaO, Fast sweeping methods for Eikonal equations on triangular meshes, SIAM Journal on Numerical Analysis, 45 (2007), pp. 83-107.

[29] E. RouY And A. Tourin, A viscosity solutions approach to shape-from-shading, SIAM Journal on Numerical Analysis, 29 (1992), pp. 867-884.

[30] J. A. Sethian, A fast marching level set method for monotonically advancing fronts, Proc. Nat. Acad. Sci., 93 (1996), pp. 1591-1595.

[31] J. A. Sethian and A. Vladimirsky, Ordered upwind methods for static Hamilton-Jacobi equations, Proc. Natl. Acad. Sci., 98 (2001), pp. 11069-11074.

[32] J.A. SEThian And A. Vladimirsky, Ordered upwind methods for static Hamilton-Jacobi equations: theory and algorithms, SIAM Journal on Numerical Analysis, 41 (2003), pp. 325-363.

[33] C.-W. SHu, High order numerical methods for time dependent Hamilton-Jacobi equations,, IMS Lecture Notes Series, Vol 11: Mathematics and Computation in Imaging Science and Information Processing, World Scientific Publishing, Singapore, to appear.

[34] C.-W. SHU, Total-variation-diminishing time discretizations, SIAM J. Sci. Statist. Comput., 9 (1988), pp. 1073-1084.

[35] C.-W. SHU AND S. Osher, Efficient Implementation of Essentially Non-Oscillatory ShockCapturing Schemes, Journal of Computational Physics, 77 (1988), pp. 439-471.

[36] Y.-H. R. Tsai, L.-T. Cheng, S. Osher And H.-K. ZhaO, Fast sweeping algorithms for a class of Hamilton-Jacobi equations, SIAM Journal on Numerical Analysis, 41 (2003), pp. 673694.

[37] J.N. TsitsikLIS, Efficient algorithms for globally optimal trajectories, IEEE Transactions on Automatic Control, 40 (1995), pp. 1528-1538.

[38] Y.-T. Zhang And C.-W. Shu, High order WENO schemes for Hamilton-Jacobi equations on triangular meshes, SIAM Journal on Scientific Computing, 24 (2003), pp. 1005-1030.

[39] Y.-T. Zhang, H.-K. ZhaO AND J. QIAN, High order fast sweeping methods for static HamiltonJacobi equations, Journal of Scientific Computing, 29 (2006), pp. 25-56.

[40] H.-K. Zhaо, A fast sweeping method for Eikonal equations, Math. Comp., 74 (2005), pp. 603627.

[41] H.-K. Zhao, S. Osher, B. Merriman And M. Kang, Implicit and non-parametric shape reconstruction from unorganized points using variational level set method, Computer Vision and Image Understanding, 80 (2000), pp. 295-319. 
